\title{
Classical and quantum physics mix
}

\author{
A neat way of talking of quantum and classical physics in the same language will be of interest in itself even if it does \\ not avoid the need for a quantum theory of gravity; but that would be a huge extra.
}

MIXING together classical mechanics and quantum mechanics is a long-standing source of embarrassment, usually arising quite early in the textbooks of quantum mechanics. There is, for example, the simplest of all the problems in quantum mechanics, that of a particle in a box. The heuristic value of this example is that it stands proxy for an electron or some other charged particle in a fixed electrostatic potential, as in the interior of a metal for example.

Beginning students are not, however, invited to wonder how the electrostatic box itself is constructed. If they were (and if the dimensions of the box were of the same order of magnitude as the wavelength of the electron), they would quickly conclude that the electrostatic field that constitutes the box can have been made only from microscopic objects, say the ions of a crystal lattice, which are themselves, in principle, also subject to the laws of quantum mechanics. So why treat the electron in a box as a quantum object, yet consider the electrostatic potential that confines it as a classical given?

Similar problems arise in more taxing fashion in textbook discussions of the problems of quantum measurement. The Heisenberg gedanken microscope used to demonstrate the inescapable uncertainty in a measurement of the position of an electron is a case in point. The idea is to estimate the position of the electron from the scattering of photons. The precision of the measurements is inevitably a function of the frequency of the photons, but the greater the frequency, the greater the momentum they will transfer to the electron, which offsets the potential benefit of working with higher frequencies.

The argument demonstrates what the authors of the textbooks want to show, that Heisenberg's Uncertainty Principle is unavoidable, but hardly anybody stops to ask whether the microscope itself is a quantum object. If, by chance, they do, the answer will be that it is a macroscopic object, the uncertainty in the position of its optical elements (calculable from the same uncertainty principle) will be shown to be much less than the uncertainty in the position of the electron, and that will be that.

Thus the world has become used to the idea that quantum physics and classical physics are a little like oil and water. They do not mix. Where some interaction is of the essence, as in the behaviour of a laser, the quantum elements (excited atoms and the NATURE · VOL 373 - 9 FEBRUARY 1995 photons whose emission is stimulated) are dealt with as such, while the structural elements of the laser (which define the shape of the resonating cavity) appear simply as parameters in the equations. This fuss does not imply that the standard treatments of mixed problems are in some sense wrong, but that they are at least inelegant and may leave people with an awkward sense of insecurity.

That seems to be part of the reason why Arlen Anderson, from Imperial College London (but also affiliated to the Newton Institute at Cambridge) has embarked on a scheme for including both quantum and classical variables in a common theory (Phys. Rev. Lett. 74, 621-625; 1995). As he puts it, he seeks to "assuage mathematical doubts about how classical and quantum variables may coexist in a single theory". But reading between the lines, the long-term objective is to find a way of handling the field theories of particle physics self-consistently against the background of a classical theory of gravity, that of Einstein's general relativity.

Anderson acknowledges earlier work in the same direction by I. V. Aleksandrov and by W. Boucher and J. Traschen, saying that the distinctive contribution is to demonstrate the 'back-reaction' of the quantum system on a classical system with which it is coupled. This effectively takes the form of an extra source of noise in the evolution of the classical system whose effect is to induce a correlation between the values of classical observables (the quantum word for 'measurable quantity' or 'variable') and those attained by the coupled quantum system.

The algebraic technology of putting together quantum and classical mechanics turns out to be simple enough, perhaps deceptively so. The starting point is the definition of a conjugate pair of variables (say $\mathbf{q}$ and $\mathbf{p}$, perhaps the position of a particle and its momentum in that direction), to which the Uncertainty Principle applies in quantum mechanics, which is that $[\mathbf{q}, \mathbf{p}]=i$ (where $[\mathbf{q}, \mathbf{p}] \equiv \mathbf{q p}-\mathbf{p q}, i$ is $\sqrt{ }(-1)$ and the units are such that $h / 2 \pi=1$ )

The classical analogue of this relationship is familiar from Hamilton's nineteenthcentury formulation of classical mechanics, which makes it possible to test whether, given one pair of conjugate variables, say $x$ and $k$, functional combinations of them, say $f(x, k)$ and $g(x, k)$, are also conjugate variables. The test is that the so-called Poisson bracket, called $\{f, g\}$, should be equal to 1 .
The Poisson bracket is defined as $\left(\partial_{x} \partial_{k} g-\partial_{k} f \partial_{x} g\right)$, where $\partial_{x}$ and $\partial_{k}$ denote partial differentiation with respect to $x$ and $k$ respectively. (Plainly the test is trivially satisfied for the original variables $x$ and $k$.)

What Anderson does is simply to put the two conditions together, arriving at a test for pairs of conjugate variables $(A$ and $B)$ with the form $[A, B]+i\{A, B\}=i$. At the most trivial level, this equation is plainly valid; if $A$ and $B$ are a conjugate pair of quantum variables, only the first term applies (and equals $i$ by definition), but if they are a classical pair, only the second is relevant and is identically true.

More generally, he defines a new bracket expression for any two variables as $[A, B]^{*}$, say, by the expression above, $[A, B]+i\{A, B\}$; he departs from others who have followed this route in the rules for evaluating $[A, B]^{*}$ for mixtures of classical and quantum variables. Following the standard procedures of quantum mechanics, there then tumbles out an expression for the time rate of change of an arbitrary function $A$ of quantum and classical variables (and in which the time is not explicit) in the familar form of $-i[A, H]^{*}$, where $H$ is the Hamiltonian of the system.

The physics of this formalism is not as obscure as it may seem (but Anderson's paper illustrates the point with two workable - and neatly worked - examples). The deficiency of representing the variables of the classical parts of a mixed system as parameters in quantum equations of motion is that they are thereby given the status of givens, and cannot be changed. But even if the classical parts are so far away from the conditions required of quantum systems that they will not, for example, be quantized in the sense that only some states are accessible to them, there is every reason why their continual interaction with a quantum part will affect the probability distribution of the states in which they may be found.

Whether these developments will solve the problem of the gravitational field is another matter. After two decades of serious attempts to produce a quantum theory of gravitation, it is forgivable that people should now be wondering whether it is really necessary to try. The clamant need for quantum gravity arises only in exceptional circumstances, at the edges of black holes and at similar exceptional locations. But to follow even Anderson's new approach, people will have to learn to do quantum mechanics in curved space-time. That will not be child's play

John Maddox 